\title{
Whole-body computed tomography in trauma patients: optimization of the patient scanning position significantly shortens examination time while maintaining diagnostic image quality
}

This article was published in the following Dove Press journal:

Therapeutics and Clinical Risk Management

\section{Tilman Hickethier ${ }^{1, *}$ \\ Kamal Mammadov',* \\ Bettina Baeßler' \\ Thorsten Lichtenstein' \\ Jochen Hinkelbein ${ }^{2}$ \\ Lucy Smith ${ }^{3}$ \\ Patrick Sven Plum ${ }^{4}$ \\ Seung-Hun Chon ${ }^{4}$ \\ David Maintz' \\ De-Hua Chang' \\ 'Department of Radiology, University Hospital of Cologne, Cologne, Germany; ${ }^{2}$ Department of Anesthesiology and Intensive Care Medicine, University Hospital of Cologne, Cologne, Germany; ${ }^{3}$ Faculty of Medicine, Memorial University of Newfoundland, St. John's, Canada; ${ }^{4}$ Department of General, Visceral and Cancer Surgery, University Hospital of Cologne, Cologne, Germany}

*These authors contributed equally to this work

\begin{abstract}
Background: The study was conducted to compare examination time and artifact vulnerability of whole-body computed tomographies (wbCTs) for trauma patients using conventional or optimized patient positioning.

Patients and methods: Examination time was measured in 100 patients scanned with conventional protocol (Group A: arms positioned alongside the body for head and neck imaging and over the head for trunk imaging) and 100 patients scanned with optimized protocol (Group B: arms flexed on a chest pillow without repositioning). Additionally, influence of two different scanning protocols on image quality in the most relevant body regions was assessed by two blinded readers.

Results: Total wbCT duration was about $35 \%$ or 3:46 min shorter in B than in A. Artifacts in aorta ( 27 vs $6 \%$ ), liver ( 40 vs $8 \%$ ) and spleen ( 27 vs $5 \%$ ) occurred significantly more often in B than in A. No incident of non-diagnostic image quality was reported, and no significant differences for lungs and spine were found.
\end{abstract}

Conclusion: An optimized wbCT positioning protocol for trauma patients allows a significant reduction of examination time while still maintaining diagnostic image quality.

Keywords: CT scan, polytrauma, acute care, time requirement, positioning

\section{Introduction}

The management of trauma patients is a highly relevant topic in health care since it is a major cause of loss of life years in developed countries, as well as a significant cost burden on health care systems. ${ }^{1}$ Multiple studies have shown that patients suffering from severe trauma should receive a whole-body computed tomography (wbCT) regardless of the specific body region injured. ${ }^{2-7}$ Additionally, it has been proven that the necessary diagnostic steps not only have to be extensive, but also urgent, because a delayed treatment can be associated with worse outcome. ${ }^{8}$ Despite the critical role of wbCT in the management of trauma patients, there is very little data on how exactly a wbCT should be performed. Currently, a variety of wbCT scanning protocols are available for use. ${ }^{9-14}$ A recent study published in May 2017, surveying Emergency Centers in Switzerland, found that the most commonly used positioning protocol among the participating hospitals has the arms of the patient positioned alongside the body for scanning the head and the neck, followed by a repositioning of their arms over the head for scanning the trunk. ${ }^{15}$ The second most commonly used protocol has the patient's arms placed on the body for the entirety of the scan without the need for repositioning.
Correspondence: De-Hua Chang Department of Radiology, University Hospital of Cologne, Kerpener-Str. 62, Cologne 50973, Germany

Tel +4922147896023

$\mathrm{Fax}+4922147882384$

Email de-hua.chang@uk-koeln.de 
This optimized positioning approach (ie, avoidance of repositioning of the patient's arms during the scan) was validated in another study, which found that positioning the patient's arms flexed over a large pillow ventrally to the chest is preferable compared to other variants without repositioning (eg, arms placed alongside the body). ${ }^{16}$

The purpose of this study was to determine the influence of these two most commonly used positioning protocols for wbCT scans of trauma patients in terms of examination time as well as the resulting image quality. To our knowledge, there is no quantitative clinical data available in regard to this topic.

\section{Material and methods}

\section{Patients characteristics and positioning during CT}

In April 2015, a new positioning protocol for patients with severe trauma, which does not require repositioning of the patient's arms during a wbCT scan, was introduced at our hospital (sample images of a scan conducted with the new protocol are given in Figure 1). In the present study, CT examinations of the first 100 patients using the new protocol (Protocol B; conducted from April to August 2015) as well as the last 100 patients with the previously used protocol (Protocol A; conducted from December 2014 to April 2015) were evaluated. Both study groups included patients with additional head and neck CT angiography (hnCTA) (A2/B2) and those without hnCTA (A1/B1) (see in the following text for details). This retrospective analysis was approved by the local ethics board of the medical faculty of University of Cologne and informed consent was waived due to the retrospective nature of the study. All accessed patient data were de-identified. Patient characteristics are summarized in Table 1.

Until April 2015, Protocol A was used in which the patient's arms are initially positioned alongside the body for scanning the head and the neck, followed by repositioning of the arms over the head for scanning the trunk. This was described as the most commonly used protocol in various trauma centers by a recent Swiss study published in May 2017. ${ }^{15}$ With the newly implemented Protocol B, both arms are flexed at the elbow and the forearms are positioned next to each other on top of a large pillow $(51 \times 23 \times 11 \mathrm{~cm})$ placed ventrally on the body at the level of the lower chest. This type of scanning protocol without the need to reposition throughout the whole scan is recommended in another Swiss study published in $2011 .{ }^{16}$

In both protocols, a contrast agent was either administered between the scan of head and neck and the scan of the trunk (Protocols A1 + B1) or between a native scan of the head and an additional hnCTA if trauma mechanism or clinical presentation of the patient indicated a possible
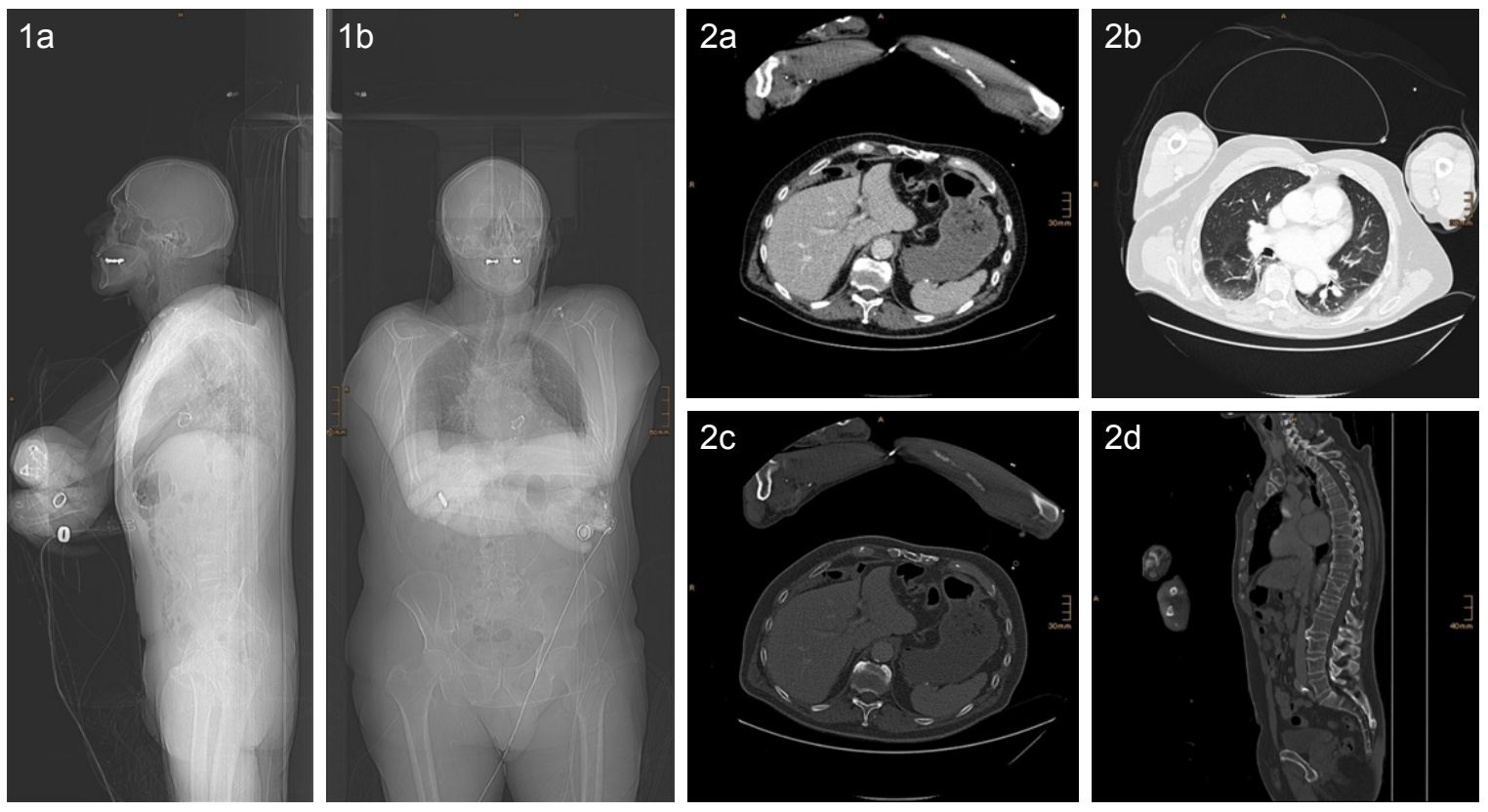

Figure I Sample images of a scan conducted with the new protocol. Ia + b: Survey scans showing arms in flexion and crossed above a pillow. 2: Reconstructed images from the trunk scan of the evaluated regions of interest.

Notes: a: Liver and spleen, b: lung, c + d: spine. For better illustration of the patient positioning, the arms were not cropped in the images for this figure. In the images used for the quality analysis, arms were cropped, respectively. 
Table I Patient characteristics

\begin{tabular}{|c|c|c|c|c|}
\hline Protocols & $\begin{array}{l}\text { Al } \\
\text { (w/o hnCTA) }\end{array}$ & $\begin{array}{l}\text { A2 } \\
\text { (w hnCTA) }\end{array}$ & $\begin{array}{l}\text { B I } \\
\text { (w/o hnCTA) }\end{array}$ & $\begin{array}{l}\text { B2 } \\
\text { (w hnCTA) }\end{array}$ \\
\hline No. of cases & 59 & 41 & 57 & 43 \\
\hline Females (\%) & 32 & 39 & 35 & 21 \\
\hline Mean patient age (years) & $38.6 \pm 14.4$ & $46.1 \pm 20.5$ & $53.4 \pm 21.3$ & $42.9 \pm 18.5$ \\
\hline Mean BMla $\left(\mathrm{kg} / \mathrm{m}^{2}\right)$ & $25.8 \pm 4.8(n=23)$ & $26.2 \pm 6.4(n=13)$ & $26.8 \pm 4.6(n=22)$ & $25.5 \pm 3.4(n=24)$ \\
\hline
\end{tabular}

Notes: anformation on BMI was not available for every patient (number of considered patients are given in brackets). Al, conventional w/o hnCTA; A2, conventional w hnCTA; BI, optimized w/o hnCTA; B2, optimized w hnCTA.

Abbreviations: w/o, without; hnCTA, head and neck computed tomography angiography; w, with; BMI, body mass index.

injury of the brain-supplying arteries (Protocols A2 + B2). Sample topogram scans of a patient with both arm positions of Protocol A1 and a patient with Protocol B1 are shown in Figure 2.

\section{CT acquisition parameters}

All examinations were performed using a 256-row CT scanner (iCT; Philips Healthcare, Cleveland, OH, USA). All patients were placed in supine position and the head was placed first on the CT table. The used scan and reconstruction parameters for the different protocols are given in Table 2.

In all patients, a $100 \mathrm{~mL}$ bolus of iso-osmolar, nonionic iodinated contrast material (Accupaque 350, $350 \mathrm{mg}$ Iohexol/mL; GE Healthcare, Braunschweig, Germany) followed by a saline flush of $25 \mathrm{~mL}$ was injected into an antecubital vein at a flow rate of $3 \mathrm{~mL} / \mathrm{s}(4 \mathrm{~mL} / \mathrm{s}$ if a hnCTA was included). In Protocols A1 and B1, the venous phase scan of thorax and abdomen (A1) or neck, thorax and abdomen (B1) was initiated 49 seconds after the attenuation in the descending thoracic aorta reached a predefined threshold of $120 \mathrm{HU}$. This delay allowed the patient to receive breathing instruction (if possible). In Protocols A2 and B2, prior to the venous phase scan of thorax and abdomen, an additional hnCTA was performed 3.8 seconds after the attenuation reached the abovementioned threshold of $120 \mathrm{HU}$.

A (statistical) hybrid iterative reconstruction algorithm (iDose4; Philips Healthcare) was used to reconstruct all images from the acquired raw data with the iteration level 4 and the following convolution kernels and window settings (width/level): Brain UB 70/35, Skull D 3000/600, Soft tissues (Neck, Thorax, Abdomen) B 360/60, Lung L 1300/-500 and Bones D 1720/530 HU. Individual adjustments of window settings were performed by the readers if required. All reconstructed images were archived in the hospital's picture
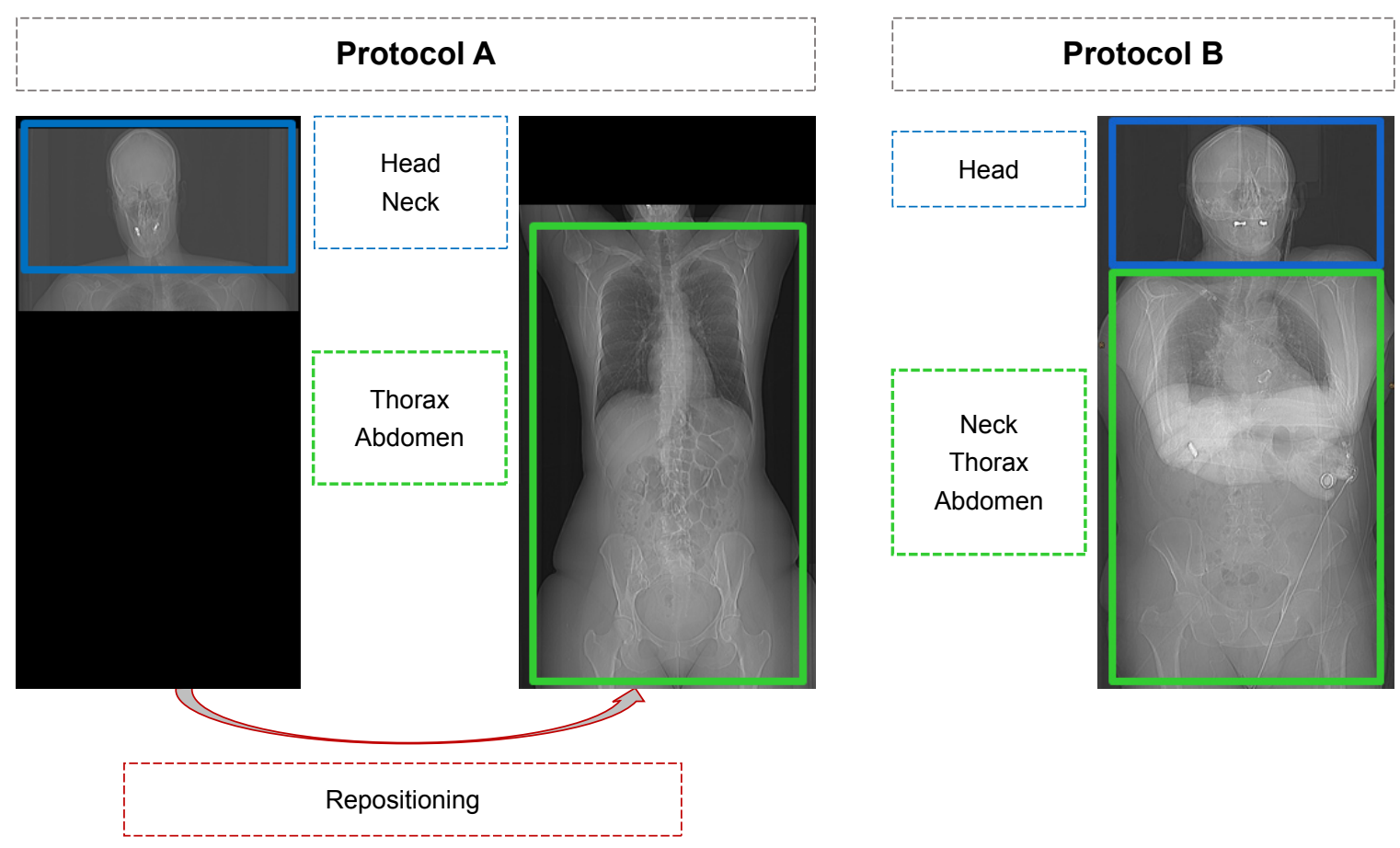

Figure 2 Sample topograms of a patient with both arm positions for Protocol AI and with the single position for Protocol BI. 


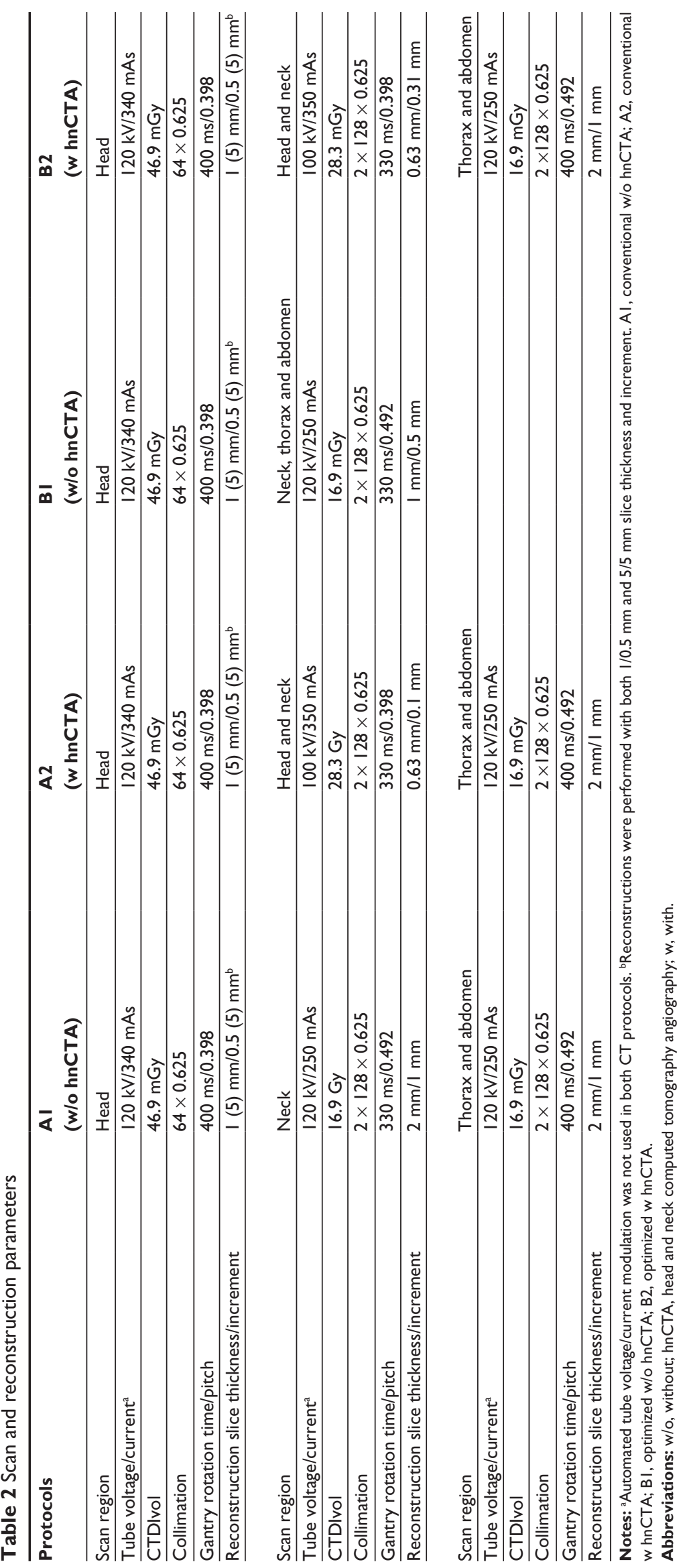



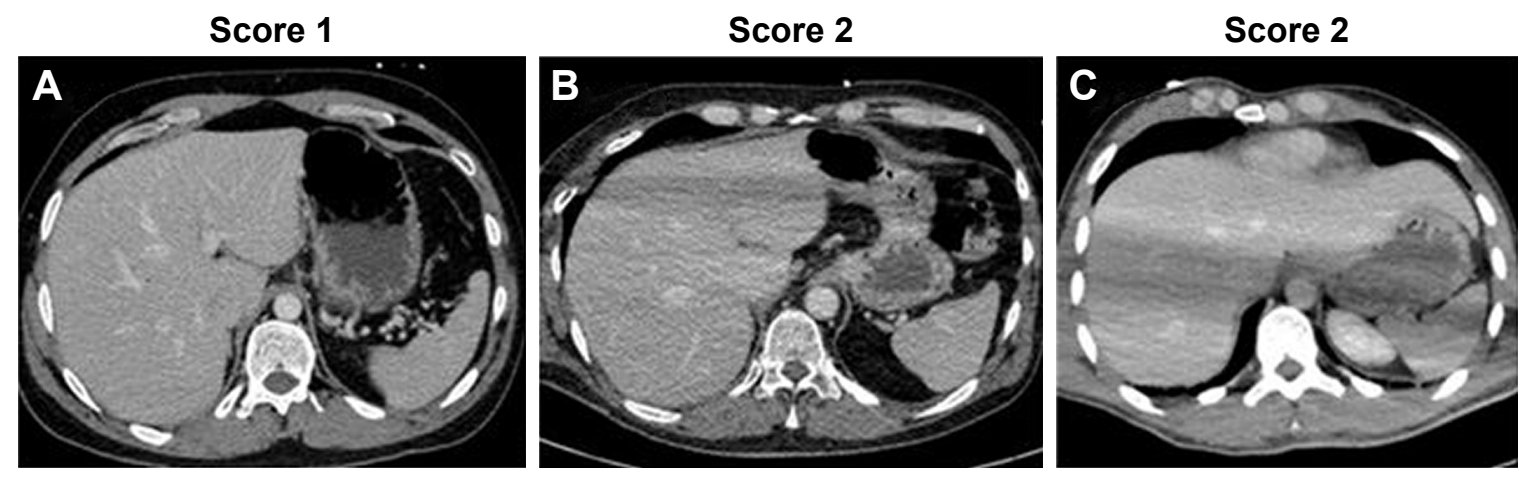

Figure 3 Sample images demonstrating the range of image quality scores in the liver: (A) no artifacts present with excellent image quality (Score I); (B, C) more pronounced artifacts present than in $(\mathbf{A})$ but with maintained diagnostic image quality (Score 2 ).

archive and communication system for further image analysis and documentary purposes.

\section{CT data analysis}

\section{Examination time assessment}

The duration of all examinations (in minutes and seconds) was determined by the automatically recorded examination times of the CT scanner and was defined as time span from the first survey scan of every examination to the last acquired image of the scan of the trunk. To additionally evaluate preparation time of the examination, the duration from the entry of the emergency room staff with the patient into the CT room to the initiation of the first survey scan was manually taken with a stopwatch in 30 patients: 10 with Protocol A, 10 with Protocol B immediately after its introduction (introduction phase) and 10 with Protocol B after it has been used for 3 months (follow-up phase).

\section{Image quality analysis}

The reconstructed images were anonymized and cropped to the anatomical regions of interest (and therefore, blinded to arm positioning) by a radiologist not involved in image analysis, who also noted whether patient positioning of Protocol B was correct, and if there were foreign objects present in the scanning area. Correct patient positioning in Protocol B is defined as arms being adequately crossed over the chest with elevated elbows (instead of just hands being folded on the upper abdomen). The presence of foreign objects is defined as inclusion of high-density objects (which can be easily identified on the surview scan) in the scanning area with relevant streak artifacts originating from them. The anonymized images were displayed in random order to two radiologists experienced in trauma imaging who were blinded to arm positioning (already discussed) as well as to each other's results. They independently assessed the subjective image quality of the liver, spleen, aorta, thoracoabdominal spine and lungs on a three-point scoring scale as follows:

- Score 1=excellent image quality, no artifacts;

- Score 2=diagnostic image quality, artifacts present;

- Score $3=$ non-diagnostic image quality due to severe artifacts.

Representative examples of images demonstrating the range of image quality scores given for the liver are presented in Figure 3.

\section{Statistical analysis}

Statistical analysis was performed in $\mathrm{R} 3.4 .0^{17}$ with RStudio 1.0.136 ${ }^{18}$ using the packages pastecs, ggplot2, ggmosaic, randomForest and irr. ${ }^{19-23}$ All continuous data are given as mean \pm standard deviation where appropriate. Testing for group differences was performed using Wilcoxon sum rank test or Welch independent $t$-test after assessing normality distribution of data. A two-tailed $p$-value of $<0.05$ was regarded as statistically significant. Concordant significant $p$-values were summarized in favor of readability. In order to evaluate the most important influencing factor on the presence of artifacts among the three parameters foreign objects, patient positioning and protocol, random forest ${ }^{24}$ and analysis of variance (ANOVA) models were fitted including interaction terms of all three parameters. Correlation between categorical variables and inter-rater agreement was assessed by calculating Kendall's Tau.

\section{Results \\ Required examination time}

The required time for a wbCT scan was significantly shorter when Protocol B was used for the examination than with Protocol A. This was found for both types of examinations, including those with an additional hnCTA $(6: 55 \pm 1: 54 \min$ vs 10:54 $\pm 2: 04 \mathrm{~min} ; p<0.01)$ and those 


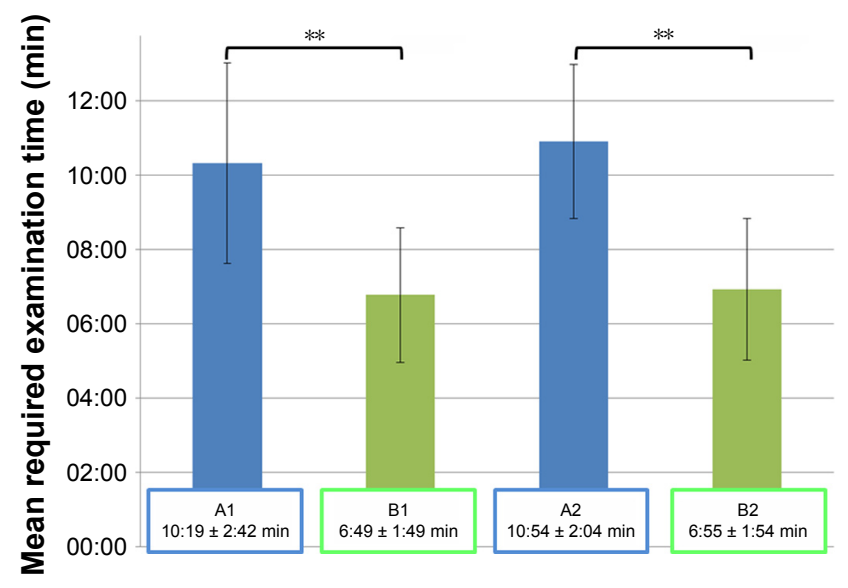

Figure 4 Mean required examination time: Protocol $B$ (no repositioning of arms) had a significantly shorter examination time (BI and B2) in comparison to Protocol A (repositioning of arms after head and neck scan) (AI and A2) in both scenarios of additional hnCTA (B2 vs $A 2$ ) and those without (BI vs $A 1$ ).

Notes: **Significant difference in examination times, Welch's $t$-test. AI, conventional w/o hnCTA; A2, conventional w hnCTA; B1, optimized w/o hnCTA; B2, optimized w hnCTA.

Abbreviation: hnCTA, head and neck computed tomography angiography.

without (6:46 $\pm 1: 49 \min$ vs 10:19 $\pm 2: 42 \mathrm{~min} ; p<0.01)$ (Figures 4 and 5).

The manually assessed preparation times with Protocol B in the introduction phase $(4: 29 \pm 1: 17 \mathrm{~min})$ are shorter than with Protocol A (4:33 $\pm 1: 15 \mathrm{~min})$, and this is especially evident after it has been used for 3 months in the follow-up phase (3:50 $\pm 0: 36 \mathrm{~min})$. The resulting differences in preparation time between Protocol A and B however, did not reach statistical significance ( $p=0.650$ for Protocol B introduction phase vs Protocol A; $p=0.305$ for Protocol B follow-up phase vs Protocol A) (Figure 6).

\section{Subjective image quality}

Interreader agreements for subjective image quality assessments were excellent between both readers with tau $(\tau)$ values of $>0.9$ for all evaluated categories except for the spleen in Protocol B1, for which it was still strongly positively correlated ( $\tau=0.792)$; therefore, only the results of the more experienced reader are given here.

In examinations with Protocol A, image quality was rated as excellent in 474 out of the 500 evaluated anatomical regions (95\%). In the other 26 anatomical regions, artifacts were present but diagnostic image quality was maintained. No relevant artifacts at all were observed in the spine. Between the other assessed regions, frequency of tolerable artifacts was similar (lung $7 \%$, aorta $6 \%$, liver $8 \%$ and spleen 5\%). There was no incident of non-diagnostic image quality.

In examinations with Protocol B, artifacts were significantly more common and occurred in 87 out of the 500 evaluated anatomical regions (17\%). Image quality was rated as excellent in 413 out of the 500 evaluated anatomical regions. Again, no relevant artifacts at all were observed in the spine and there was also no incident of non-diagnostic image quality. Artifacts occurrences in scans with Protocol B were unevenly distributed and most often affected the liver ( $40 \%$ of examinations). They were also common in the aorta and the spleen, while they were hardly seen in the lungs (27\% and $18 \%$ vs $2 \%$ of examinations).

Image quality of the spleen, liver and aorta was rated significantly higher in Protocol A when compared to Protocol B (each, $p<0.01$ ). For lungs and spine, there were no significant

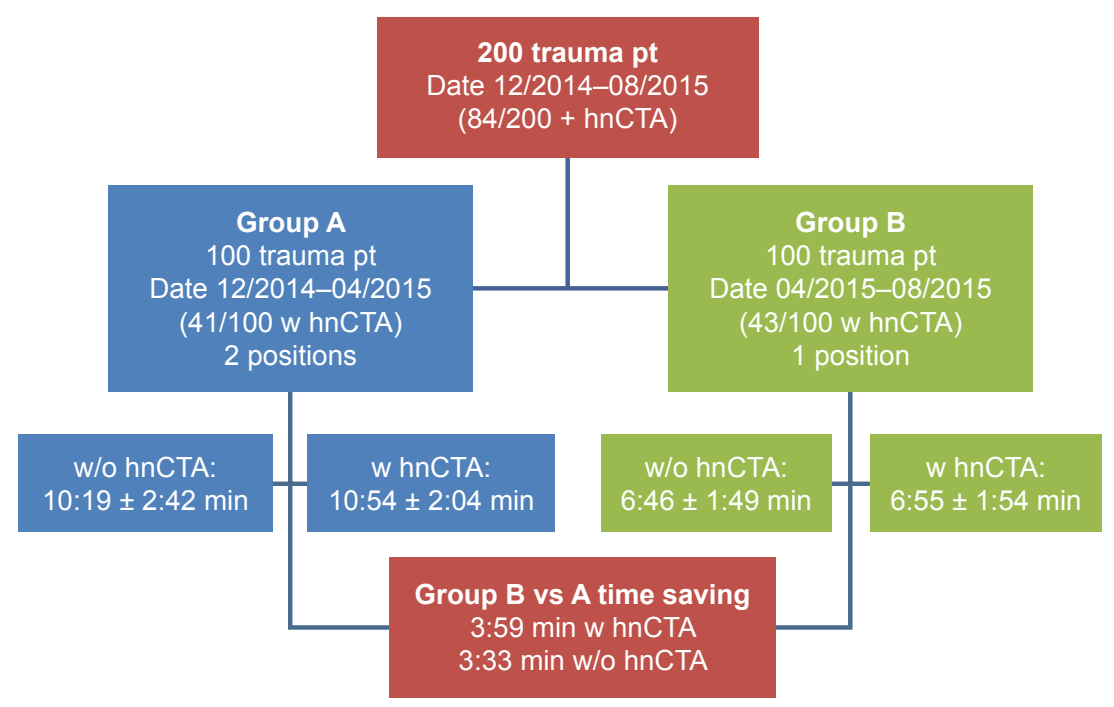

Figure 5 Flowchart of patient distribution in both groups and respective scanning time.

Abbreviations: pt, patients; hnCTA, head and neck computed tomography angiography; w/o, without; w, with. 


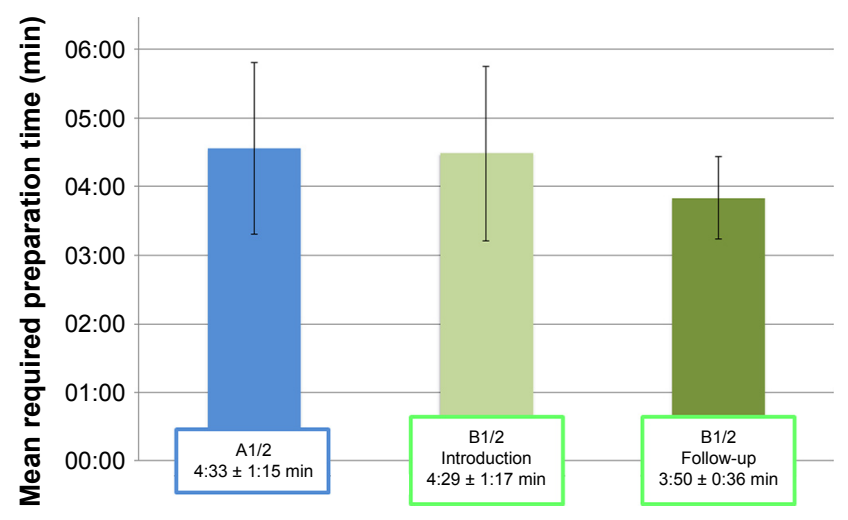

Figure 6 Mean required preparation times: preparation tends to be faster with Protocol B than with Protocol A, which, however, was not statistically significant. Note: Al, conventional w/o hnCTA; A2, conventional w hnCTA; BI, optimized w/o hnCTA; B2, optimized w hnCTA.

differences in image quality ratings $(p>0.05)$. Subjective image quality results are summarized in Table 3 and illustrated in Figure 7.

\section{Influence of foreign objects and quality of patient positioning on image quality}

Foreign objects were present with Protocol A and B within the scanning area in about every $1 / 5$ examination (18 in Protocol A and 19 in Protocol B out of the respective 100 evaluated examinations; $p>0.05$ ). In Protocol A, no artifacts occurred in any of the examined anatomical regions in the absence of foreign objects. In Protocol B, there were significantly

Table 3 Subjective image quality results

\begin{tabular}{cll}
\hline $\begin{array}{l}\text { Organ region } \\
\text { scores }\end{array}$ & $\mathbf{A I + 2}$ & $\mathbf{B ~ I + 2}$ \\
\hline Lung & 93 & 98 \\
I & 7 & 2 \\
2 & 0 & 0 \\
3 & & \\
Aorta & 94 & 73 \\
I & 6 & 27 \\
2 & 0 & 0 \\
3 & & \\
Liver & 92 & 60 \\
I & 8 & 40 \\
2 & 0 & 0 \\
3 & & 82 \\
Spleen & 95 & 18 \\
I & 5 & 0 \\
2 & 0 & 100 \\
3 & 100 & 0 \\
Spine & 0 & 0 \\
I & 0 & \\
2 & & \\
3 & &
\end{tabular}

Notes: Number of scores given for the respective organ regions (I, excellent image quality; 2, moderate image quality; 3 , non-diagnostic image quality). AI, conventional w/o hnCTA; A2, conventional w hnCTA; BI, optimized w/o hnCTA; B2, optimized w hnCTA. less artifacts for the aorta, liver and spleen if no foreign objects were present ( 23 vs $42 \%, 32$ vs $74 \%, 14$ vs $37 \%$; all $p<0.05)$. For the lungs, likewise no artifacts occurred in the absence of foreign objects. Association between presence of foreign objects and frequency of artifacts is illustrated in Figure 8 and an example of an artifact presence is given in Figure 9.

For Protocol B, the occurrence of artifacts was also influenced by the quality of patient positioning. If patient positioning was incorrect (ie, arms were not crossed over the chest with hands just folded on the upper abdomen), there were significantly more artifacts present in the aorta, liver and spleen than if arm positioning was correct (75 vs $29 \%$ and 50 vs $8 \%$; both $p<0.01$ ). For lung and aorta, there was also a trend toward increased artifacts if patient positioning was incorrect, which, however, did not reach statistical significance ( 4 vs $1 \%, p=0.38$ and 33 vs $25 \%$, $p=0.42$ ). Association between the quality of patient positioning and frequency of artifacts is illustrated in Figure 10 and an example is also given in Figure 9. Consequently, random forest and ANOVA models were used to reveal the most important cause for the presence of artifacts among the three parameters: 1) presence of foreign objects, 2) patient positioning faults and 3) positioning protocol used. The presence of foreign objects was found to be the most important factor according to the Gini index for all anatomic regions except for the spleen, for which incorrect patient positioning was more important than the presence of foreign objects (Figure 11). In concordance, ANOVAs showed highest levels of significance for the presence of foreign objects as single predictor for all anatomic regions except for the spleen. Significant interactions between the three parameters were observed in ANOVA models, although none of these showed a higher level of significance than single parameters alone.

\section{Discussion}

Our study showed that an optimized positioning protocol for trauma patients allows the continuous acquisition of a wbCT with a significantly reduced examination time, resulting in about $30 \%$ scanning time reduction compared to the common alternative protocol that requires the repositioning of the patients' arms between the acquisition of the head and neck and the trunk scans. This reduction in time requirement is found in examinations including additional hnCTA scans and in those without. Although the number of manually assessed pre-scan preparation times is limited, there is no evidence suggesting that the patient preparation prior to the 


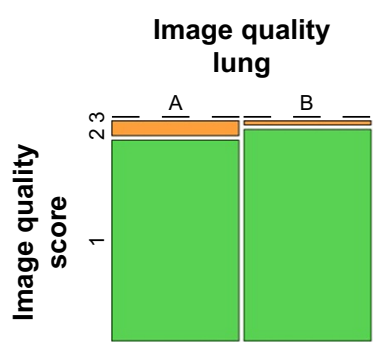

Protocol

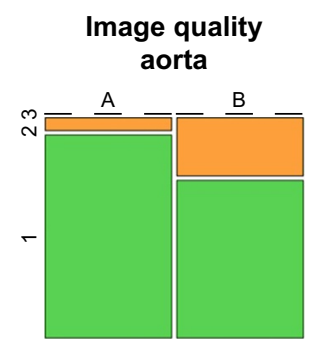

Protocol

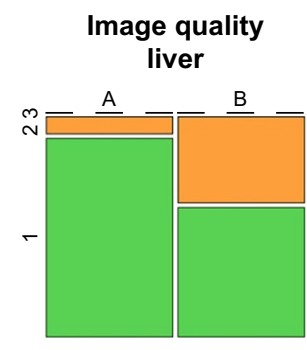

Protocol

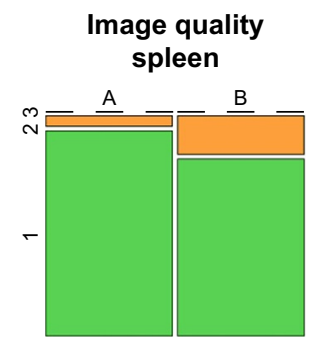

Protocol
Image quality vertebral column

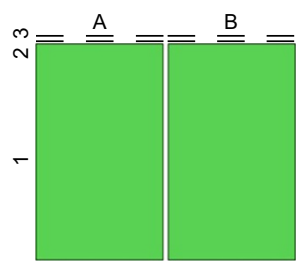

Protocol

Figure 7 Mosaic plots illustrating the frequency of artifacts with respect to the different anatomic regions and Protocols $A$ and $B$ used. Notes: A, conventional; B, optimized.

scan with the optimized positioning protocol might be more time consuming; conversely, there is a detectable trend on shorter preparation times with the optimized protocol.

On the other hand, evaluation of subjective image quality revealed that artifacts occurred significantly more often when the optimized positioning protocol was used. This led to a decline in image quality, especially in regard to the spleen but also concerning the liver and the aorta. It is worth noting, however, that no incidents of non-diagnostic image quality have been reported. For the lungs and the spine, no significant differences were found in regard to image quality.

Further analysis revealed that the presence of foreign objects in the scanning area is the most important cause for a decline in the image quality rather than the difference in positioning protocol. In addition, incorrect patient positioning with the optimized protocol is responsible for a relevant number of artifacts, and this was subsequently found to be the most important factor with respect to image quality from the spleen region.
The results of our study provide evidence that in a time-critical setting, the optimized positioning protocol as described can be used to obtain a significantly faster wbCT acquisition for trauma patients while still maintaining diagnostic image quality. This is especially true if caution is given to the removal of foreign objects and correct patient positioning. This approach is also recommended in the latest guidelines of the German Roentgen Society for imaging of severely injured trauma patients. ${ }^{25}$ On the other hand, however, our study also demonstrated that this shorter examination time may come at the expense of a slightly decreased image quality, which has been validated in a parallel study for this particular patient positioning. ${ }^{16}$ In addition, consideration should be given for when automated exposure control is used (which was not the case in our study); the inclusion of the arms in the scanning area can potentially lead to a significant increase in radiation dose (ranging from 29\% to 45\%) as described by earlier studies. ${ }^{12,16,26}$ For these reasons, the German Roentgen Society recommends the use of a protocol

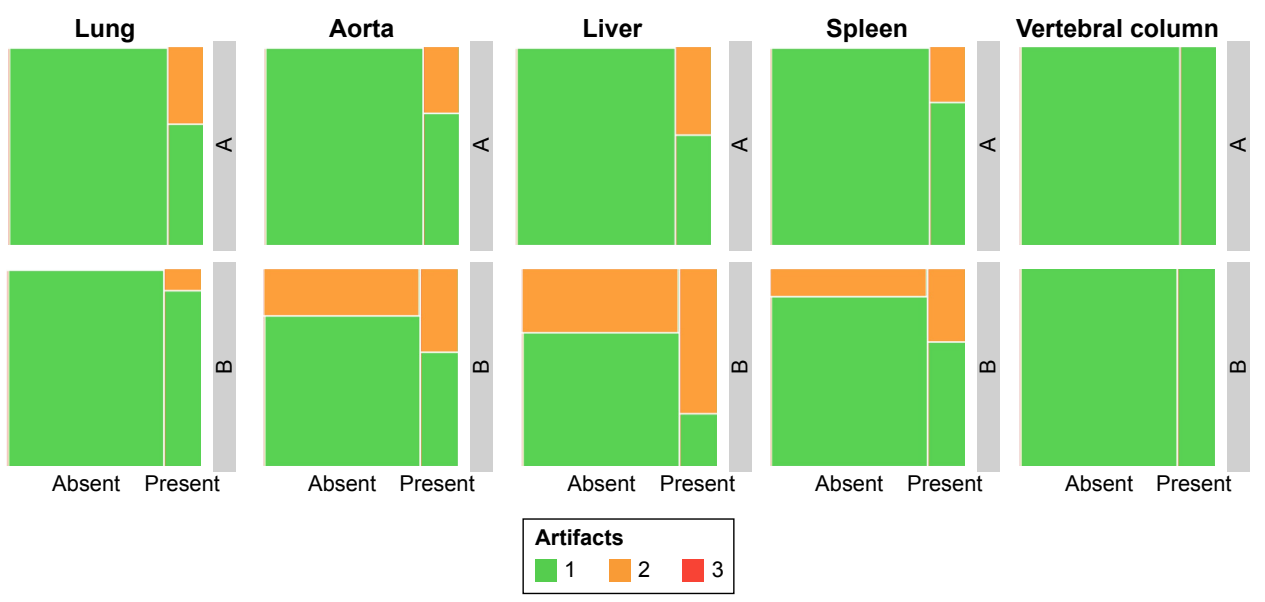

Figure 8 Mosaic plots illustrating the frequency of artifacts in regard to the presence of foreign objects (upper row Protocol A, lower row Protocol B). Notes: A, conventional; B, optimized. 

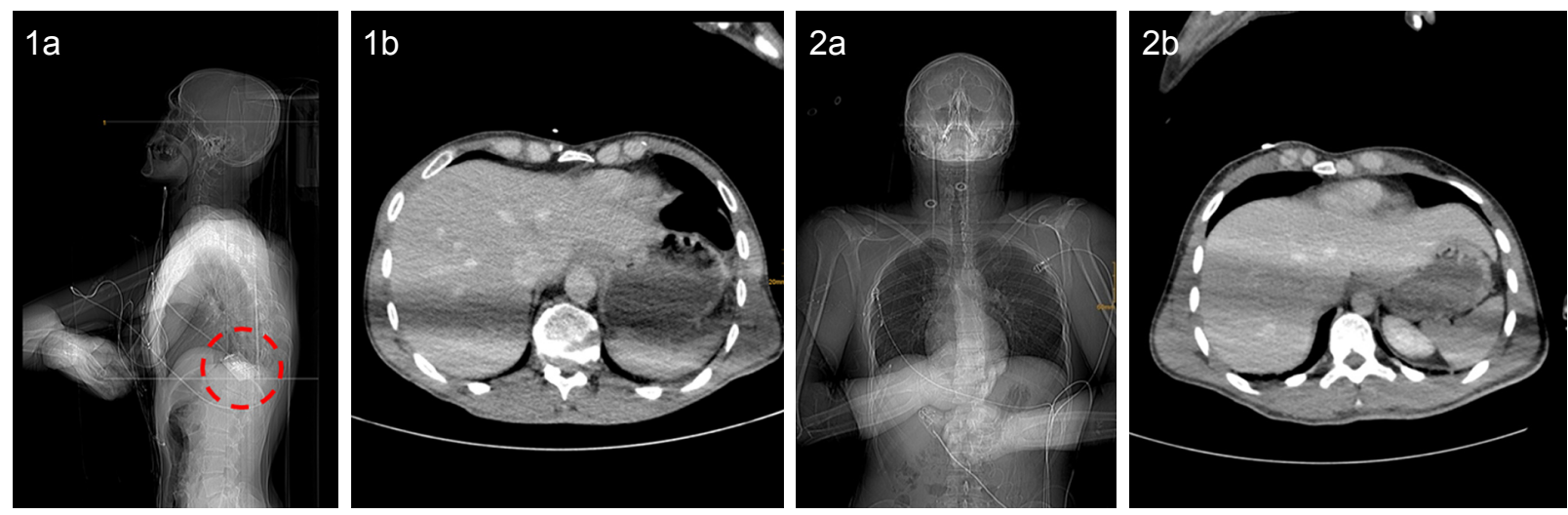

Figure 9 Sample images demonstrating artifacts due to a foreign object ( $\mathrm{la}$ and b; pulsoxymetry connector, marked with dashed circle) as well as due to incorrect positioning ( $2 \mathrm{a}$ and $\mathrm{b}$; note that arms were not crossed over the chest, with hands just folded on the upper abdomen).

with repositioning of the arms for a wbCT in more stable trauma patients under less time constrains for the benefit of image quality and minimization of radiation dose. ${ }^{25}$ The recommendations from the German Roentgen Society are in agreement with the results of our investigation.

\section{Limitations}

Our study has the limitation that despite having cropped the images to the regions of interest, the readers might have been unblinded to the arm positioning by specific beam hardening artifacts if the arms were placed within the scanning area (even if the arms themselves were not included in the actual images); this issue has been reported in previous similar studies. ${ }^{12,16,27}$ There were also incidents of which artifacts originated from foreign objects, and this type of artifacts did not give away clues to the arm position. Regarding the preparation time (duration from the entry of the emergency room staff with the patient to the initiation of the first survey scan), it has to be noted that only a small proportion of all cases was recorded. This might have led to a selection bias. In addition, the retrospective design of our study in itself is a limitation; patient characteristics, however, were fairly comparable between both groups. Another limitation is that the influence of the injury severity was not taken into account in this study, which might have been different among the two study groups. Realistically, injury severity would only be a minor limitation because the two primarily evaluated parameters, the duration of the CT scan itself and the image quality, usually do not strongly depend on the patient condition.

\section{Conclusion}

Our study has statistically proven that the continuous acquisition of a wbCT for trauma patients can be more efficiently performed with the patients' arms placed on a pillow ventrally to the chest without the need for repositioning between the acquisition of the head and neck and the trunk scans. This optimized patient scanning position leads to about $30 \%$ shorter examination time and still maintains diagnostic image quality. The efficiency gain from this optimized patient scanning protocol does come at the expense of slightly decreased
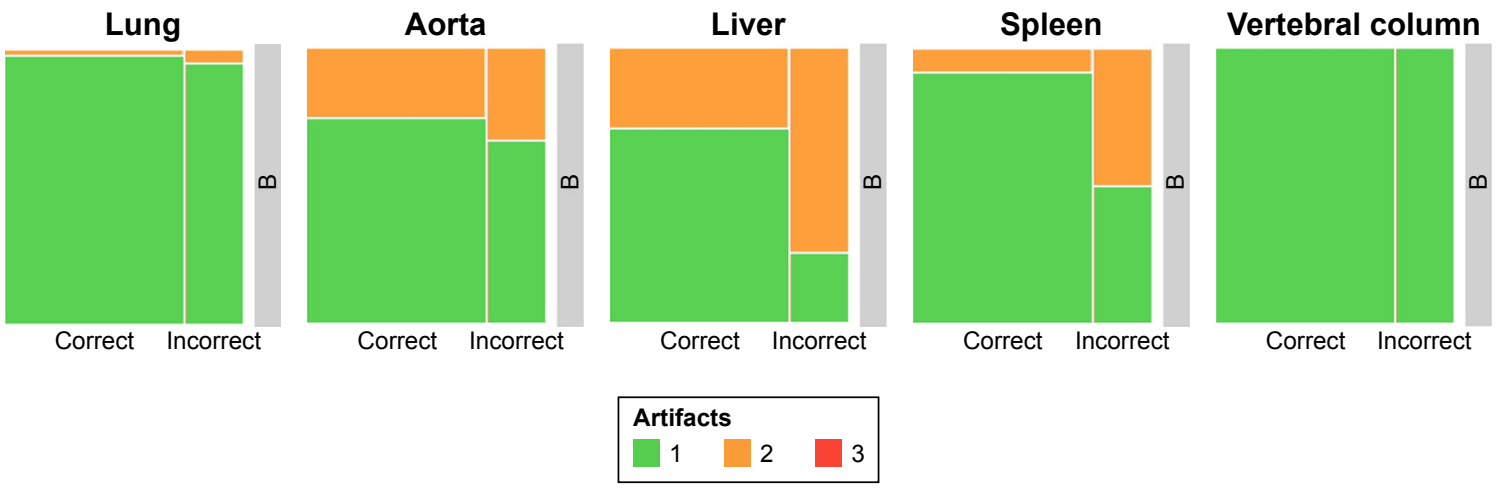

Figure 10 Mosaic plots illustrating the frequency of artifacts in Protocol B depending on the quality of patient positioning. Notes: A, conventional; B, optimized. 

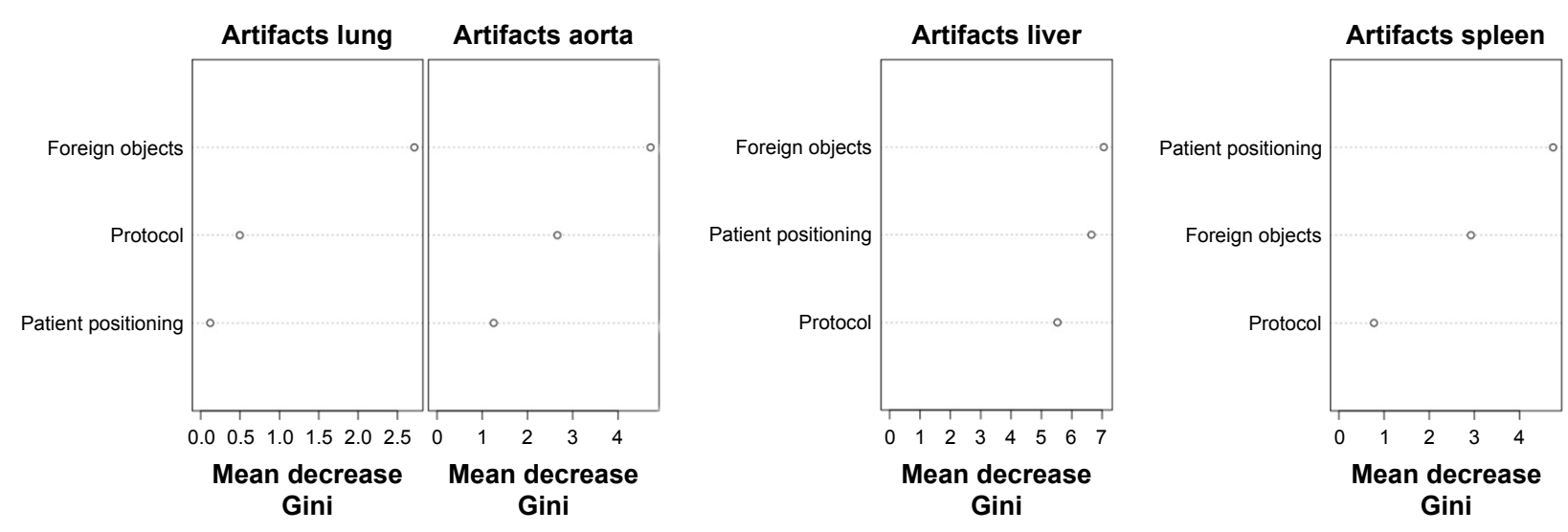

Figure I I Variable importance measures from random forest models indicating the mean decrease Gini indices for the three parameters predicting the presence of artifacts. Highest Gini indices indicate most important features.

image quality overall; nevertheless, we are also able to demonstrate there are other confounding factors such as the presence of foreign objects and incorrect positioning of the patient that contributed to the production of artifacts. It is worth emphasizing that diagnostic value was still maintained in every scan from the optimized positioning protocol even with artifacts present; hence, we can state with confidence that the optimized positioning protocol is a reliable and more efficient way of conducting wbCT in trauma patients. In consideration of optimal image quality and minimization of radiation exposure, this protocol is primarily recommended for time-critical situations.

\section{Disclosure}

The authors report no conflicts of interest in this work.

\section{References}

1. MacKenzie EJ, Morris JA Jr, Smith GS, Fahey M. Acute hospital costs of trauma in the United States: implications for regionalized systems of care. J Trauma. 1990;30(9):1096-1101.

2. Huber-Wagner S, Lefering R, Qvick LM, et al. Effect of whole-body $\mathrm{CT}$ during trauma resuscitation on survival: a retrospective, multicentre study. Lancet. 2009;373(9673):1455-1461.

3. Becker CD, Poletti PA. The trauma concept: the role of MDCT in the diagnosis and management of visceral injuries. Eur Radiol. 2005; 15 (Suppl 4) :D105-D109.

4. Broder J, Warshauer DM. Increasing utilization of computed tomography in the adult emergency department, 2000-2005. Emerg Radiol. 2006;13(1):25-30.

5. Leidner B, Adiels M, Aspelin P, Gullstrand P, Wallén S. Standardized CT examination of the multitraumatized patient. Eur Radiol. 1998;8(9): 1630-1638.

6. Novelline RA, Rhea JT, Rao PM, Stuk JL. Helical CT in emergency radiology. Radiology. 1999;213(2):321-339.

7. Huber-Wagner S, Biberthaler P, Häberle S, et al; TraumaRegister DGU. Whole-body CT in haemodynamically unstable severely injured patients - a retrospective, multicentre study. PLoS One. 2013;8(7): e68880.

8. Clarke JR, Trooskin SZ, Doshi PJ, Greenwald L, Mode CJ. Time to laparotomy for intra-abdominal bleeding from trauma does affect survival for delays up to 90 minutes. $J$ Trauma. 2002;52(3):420-425.
9. Heyer CM, Rduch G, Kagel T, et al. Prospektive, randomisierte Evaluation eines modifizierten Mehrdetektor-CT-Protokolls in der Initialdiagnostik beim Polytrauma [Prospective randomized trial of a modified standard multislice CT protocol for the evaluation of multiple trauma patients]. Rofo. 2005;177(2):242-249. German [with English abstract].

10. Loewenhardt B, Buhl M, Gries A, et al. Radiation exposure in wholebody computed tomography of multiple trauma patients: bearing devices and patient positioning. Injury. 2012;43(1):67-72.

11. Sedlic A, Chingkoe CM, Tso DK, Galea-Soler S, Nicolaou S. Rapid imaging protocol in trauma: a whole-body dual-source CT scan. Emerg Radiol. 2013;20(5):401-408.

12. Brink M, de Lange F, Oostveen LJ, et al. Arm raising at exposurecontrolled multidetector trauma $\mathrm{CT}$ of thoracoabdominal region: higher image quality, lower radiation dose. Radiology. 2008;249(2): 661-670.

13. Smith CM, Mason S. The use of whole-body CT for trauma patients: survey of UK emergency departments. Emerg Med J. 2012;29(8): 630-634.

14. Wiklund E, Koskinen SK, Linder F, Åslund PE, Eklöf H. Whole body computed tomography for trauma patients in the Nordic countries 2014: survey shows significant differences and a need for common guidelines. Acta Radiol. 2016;57(6):750-757.

15. Hinzpeter R, Boehm T, Boll D, et al. Imaging algorithms and CT protocols in trauma patients: survey of Swiss emergency centers. Eur Radiol. 2017;27(5):1922-1928.

16. Karlo C, Gnannt R, Frauenfelder T, et al. Whole-body CT in polytrauma patients: effect of arm positioning on thoracic and abdominal image quality. Emerg Radiol. 2011;18(4):285-293.

17. R Core Team. R: A language and environment for statistical computing (R Foundation for Statistical Computing). Available from: https:// www.r-project.org/. Accessed September 12, 2015.

18. RStudio. RStudio. Boston, MA: RStudio, Inc.; 2012. Available from: https://www.r-project.org/. Accessed September 12, 2015.

19. Grosjean P, Ibanez F, Etienne M. Pastecs: Package for Analysis of Space-Time Ecological Series. Available from: https://github.com/ phgrosjean/pastecs. Accessed April 7, 2018.

20. ggplot2. Available from: http://ggplot2.org/. Accessed October 27, 2016.

21. Jeppson H, Hofmann H, Cook D, Wickham H. Ggmosaic: Mosaic Plots in the "ggplot2" Framework; 2017. Available from: https://cran.rproject.org/web/packages/ggmosaic/index.html. Accessed November 5, 2017.

22. Liaw A, Wiener M. Classification and Regression by randomForest. R News. 2002;2(3):18-22

23. Gamer M, Lemon J, Singh IFP. Irr: Various Coefficients of Interrater Reliability and Agreement; 2012. Available from: https://cran.r-project. org/web/packages/irr/index.html. Accessed November 5, 2017. 
24. Breiman L. Random forests. Mach Learn. 2001;45(1):5-32.

25. Protokollempfehlungen der AG Bildgebende Verfahren des Bewegungsapparats (AG BVB) zur Ganzkörper-CT mehrfach verletzter bzw. polytraumatisierter Patienten [Protocol recommendations of the AG Imaging procedures of the musculoskeletal system (AG BVB) for whole-body CT of multiple injured or polytraumatized patients]. Rofo. 2017;189(10):1002-1006. German [with English abstract].
26. Bayer J, Pache G, Strohm PC, et al. Influence of arm positioning on radiation dose for whole body computed tomography in trauma patients. J Trauma. 2011;70(4):900-905.

27. Schertler T, Glücker T, Wildermuth S, Jungius KP, Marincek B, Boehm T. Comparison of retrospectively ECG-gated and nongated MDCT of the chest in an emergency setting regarding workflow, image quality, and diagnostic certainty. Emerg Radiol. 2005;12(1-2):19-29.

\section{Publish your work in this journal}

Therapeutics and Clinical Risk Management is an international, peerreviewed journal of clinical therapeutics and risk management, focusing on concise rapid reporting of clinical studies in all therapeutic areas, outcomes, safety, and programs for the effective, safe, and sustained use of medicines. This journal is indexed on PubMed Central, CAS,
EMBase, Scopus and the Elsevier Bibliographic databases. The manuscript management system is completely online and includes a very quick and fair peer-review system, which is all easy to use. Visit http://www.dovepress.com/testimonials.php to read real quotes from published authors.

Submit your manuscript here: http://www.dovepress.com/therapeutics-and-clinical-risk-management-journal 